\title{
Distribution and population size of Black Hole Binaries in the Galaxy
}

\section{E.J.A. Meurs ${ }^{1}$}

School of Cosmic Physics, DIAS, and School of Physical Sciences, DCU

Glasnevin, Dublin 9, Ireland

E-mail: ejameurs@gmail.com

\section{G. O'Halloran}

School of Physical Sciences, Dublin City University

Glasnevin, Dublin 9, Ireland

E-mail: gavin. ohalloran4@mail.dcu.ie

\section{Norci}

School of Physical Sciences and NCPST, Dublin City University

Glasnevin, Dublin 9, Ireland

E-mail: laura.norci@gmail.com

\section{P.J. Kavanagh}

School of Physical Sciences and NCPST, Dublin City University

Glasnevin, Dublin 9, Ireland

E-mail: patrick.kavanagh8@mail.dcu.ie

We present a map of Black Hole Binaries (BHBs) in our Galaxy, based on a comprehensive review of their distances to the Sun and utilising a recent Milky Way spiral structure result derived from Spitzer data. The observed Galactic BHB distribution is used to obtain a population size of 1800 objects, assuming that they are distributed like Population II objects, in view of generally low mass stellar companions. When recurrence times and unconfirmed, candidate BHBs are taken into account, the total number may increase to 10,000 objects.

8th INTEGRAL Workshop "The Restless Gamma-ray Universe"

Dublin, Ireland

September 27-30, 2010

\footnotetext{
$1 \quad$ Speaker
} 


\section{The Galactic BHB population}

While X-ray binaries most often contain Neutron Stars, some of these systems have a Black Hole as collapsed component. Usually the latter binaries feature a low mass normal stellar companion. Estimates of the total number of Black Hole Binaries (BHBs) in our Galaxy vary from author to author.

\section{Confirmed BHBs in the Galaxy}

A recent overview of confirmed BHBs by Casares (2007) lists 17 such objects in the Galaxy (leaving out BW Cir, that has an abnormally big distance), comprising 16 Low Mass X-ray Binaries (LMXBs) and 1 High Mass X-ray Binary (Cyg X-1). We have searched extensively the literature for the best available distance estimates for these BHBs (details to follow in Meurs et al., in preparation). Table 1 presents these 17 objects with their adopted distances, plus some other relevant data from Casares (2007). Their Galactic distribution around the Sun is displayed in Figure 1.

Table 1: Confirmed Black Hole Binaries in the Galaxy

$\begin{array}{lcllc}\text { BHB } & \begin{array}{c}\text { Distance } \\ (\mathrm{kpc})\end{array} & \begin{array}{l}P_{\text {orb }} \\ (\text { day })\end{array} & \text { Companion } & \begin{array}{c}M_{B H} \\ \left(M_{\text {Sun }}\right)\end{array} \\ \text { 4U1543-475 } & 7.5 & 1.125 & \text { A2V } & 9.4 \\ \text { A0620-003 } & 1.2 & 0.325 & \text { K4V } & 11 \\ \text { GROJ0422+32 } & 2.65 & 0.212 & \text { M2V } & 4 \\ \text { GROJ1655-40 } & 1 & 2.620 & \text { F3/5IV } & 6.3 \\ \text { GRS1009-45 } & 3 & 0.283 & \text { K7/M0V } & 5.2 \\ \text { GRS1915+105 } & 12 & 33.5 & \text { K/MIII } & 14 \\ \text { GS1124-684 } & 5.1 & 0.433 & \text { K3/5V } & 7.0 \\ \text { GS2000+25 } & 2 & 0.345 & \text { K3/7V } & 7.5 \\ \text { GX339-4 } & 7.6 & 1.754 & & \\ \text { H1705-250 } & 3 & 0.520 & \text { K3/7V } & 6 \\ \text { V404Cyg } & 2.39 & 6.471 & \text { K0IV } & 12 \\ \text { XTEJ1118+480 } & 1.72 & 0.171 & \text { K5/M0V } & 6.8 \\ \text { XTEJ1550-564 } & 5.3 & 1.542 & \text { G8/K8IV } & 9.6 \\ \text { XTE1650-500 } & 2.6 & 0.321 & \text { K4V } & \\ \text { XTE1819-254 } & 0.55 & 3.13 & \text { B9III } & 7.1 \\ \text { XTE1859+226 } & 11 & 0.382 & & \\ \text { Cyg X-1 } & 2.5 & 5.600 & \text { O9.7Iab } & 10\end{array}$




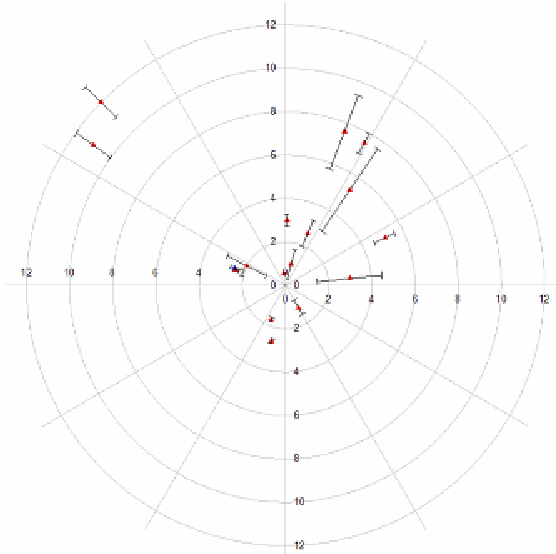

Figure 1. Black Hole Binaries around the Sun, projected on the Galactic Plane. The radial coordinate is in kpc. Red triangles: LMXBs; blue triangle: the HMXB Cyg X-1.

\section{BHB map of the Galaxy}

To assess the distribution of the BHBs in our Galaxy (Table 1), we have selected the probably most recent view of the Galaxy, obtained from Spitzer observations (Spitzermap 2008). The interpretative Spitzer map shows the Galaxy with two major arms (Scutum-Centaurus and Perseus) and two minor arms (Norma and Sagittarius). The BHBs are overlaid on this map in Figure 2, on a polar grid centered on the Sun with radius in lightyears. Figure 3 presents a Sun-centered polar grid with radius scale in kpc. The spiral arms of Figure 2 have been approximated by logarithmic spirals in this picture.

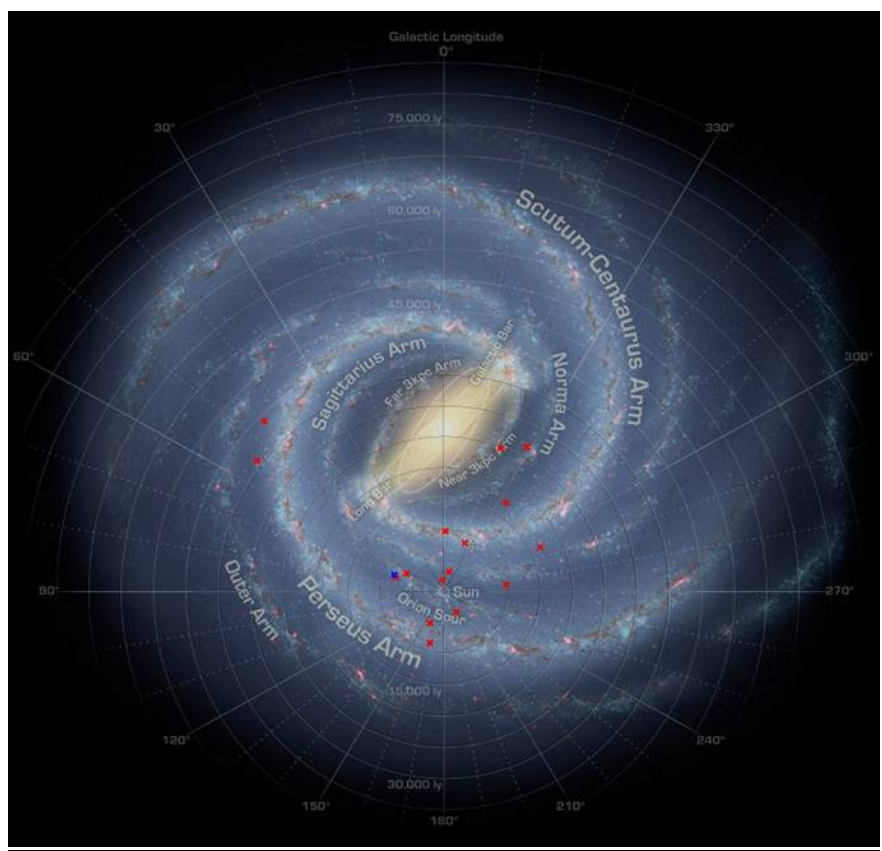

Figure 2. Spitzer map of Galaxy with BHBs from Figure 1 overlaid. Radial coordinate in lightyears. 


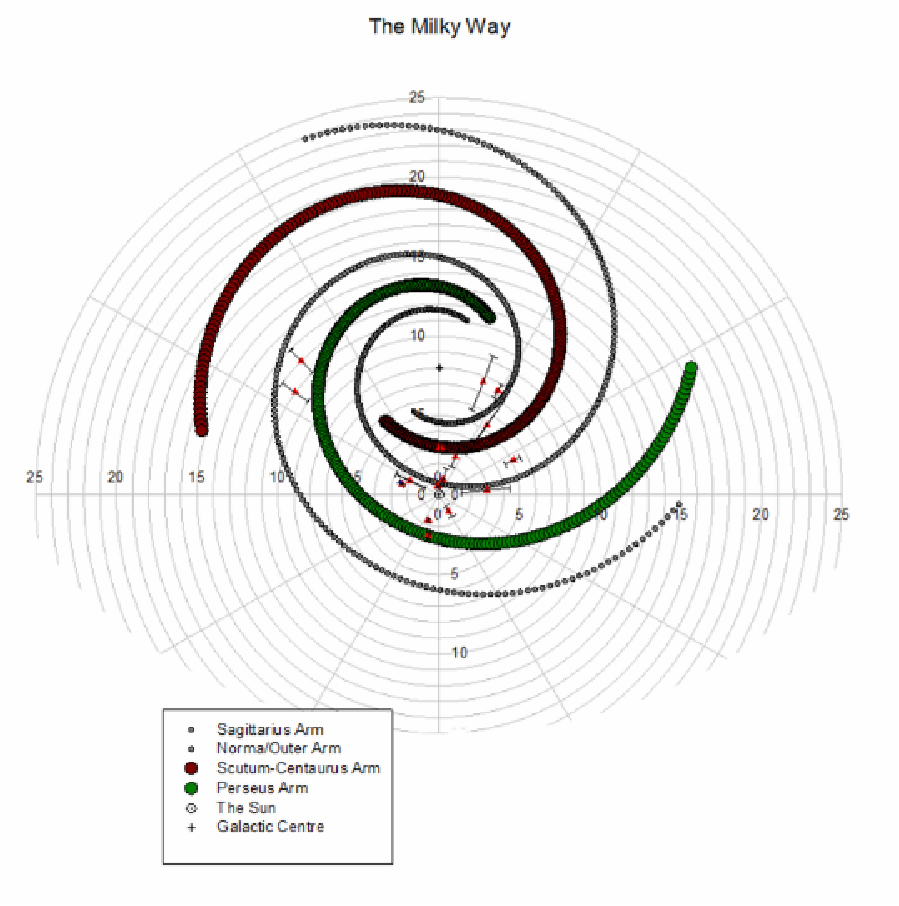

Figure 3. BHBs around the Sun with spiral arms of Galaxy indicated (based on Figure 2). Radial coordinate in $\mathrm{kpc}$.

\section{The Galactic distribution of BHBs}

Most of the BHBs in Table 1 are found close to the Sun. Figure 4 gives the number of LMXBs observed (that is, 16 of the 17 in Table 1), in increasing annuli of 1 $\mathrm{kpc}$ around the Sun. The initial increase in number of sources is almost as for a homogeneous surface distribution (viz. 1-3-5, allowing a $\sqrt{ } \mathrm{N}$ statistical error in the first bin).

No. of Sources in Concentric Annuli Around the Sun

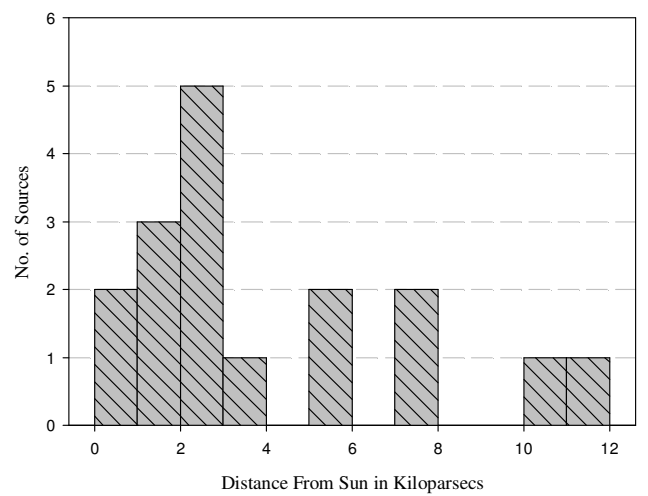

Figure 4. Number of BHBs in $1 \mathrm{kpc}$ annuli around the Sun. 
The great majority of the BHBs in Table 1 is located in the Galactic Centre half of the sky, only 3 are in the Anti-Centre half. This is an effect of the greater stellar density, increasing towards the Galactic Centre within the Solar circle and of the greater area covered in our Galaxy. If we assume that the 10 LMXB BHBs within $3 \mathrm{kpc}$ from the Sun form a fairly complete sample and that stellar numbers increase towards the Galactic Centre as does the light for Population II objects (as the companion stars are mostly long-living low mass stars), then we can obtain an estimate of the total number of BHBs in the Galaxy. Figure 5 depicts the dependence of BHBs on Galactocentric radius, for an $R^{1 / 4}$ distribution as is appropriate for Population II. The result for the total number of BHBs is a total of 1800 such objects in the Galaxy.

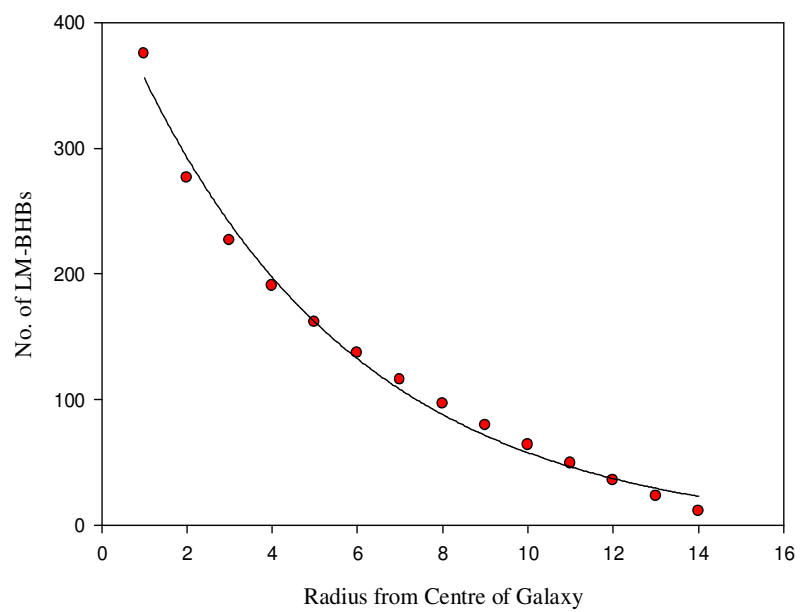

Figure 5. Estimated number of BHBs in the Galaxy as function of Galactocentric radius. The red points result from a simple numerical procedure, the fully drawn curve shows the proper $R^{1 / 4}$ dependence that was assumed.

Most of the sky has been monitored at times over the past ca. 40 years, during which the $16 \mathrm{LMXB}$ BHBs have become known. If it is assumed that BHBs have recurrence times of about $100 \mathrm{yr}$ (White \& Van Paradijs 1996), then the total number of BHBs in the Galaxy could be 4500 . A further increase by a factor of 2 could be required in view of the number of candidate BHBs that is being considered (e.g. McClintock \& Remillard 2006), in that case leading to a grand total of $\sim 10,000$. For the candidate BHBs, confirmation observations may have been prevented as recurrence times left them dormant at subsequent observing campaigns.

For comparison, total numbers that have been quoted in the literature include: about 500 (Romani 1992), based on binary evolution considerations; between a few hundred and a few thousand (Tanaka \& Lewin 1995), looking at observed numbers and recurrence times; about 500 (White \& Van Paradijs 1996), from the Galactic Zdistribution of the objects (but using clearly less complete data than available now); and about 15,000 (Sadowski et al. 2008), from population synthesis calculations. 


\section{References}

[1] J. Casares, Observational evidence for stellar-mass black holes, IAUS 238, 3 (2007)

[2] J.E. McClintock, R.A. Remillard, Black hole binaries, in Compact stellar X-ray sources, W. Lewin and M. van der Klis (Eds), Cambridge, p. 157 (2006)

[3] R.W. Romani, Populations of low-mass black hole binaries, ApJ 399, 621 (1992)

[4] A. Sadowski, J. Ziółkowski, K. Belczyński, T. Bulik, Calculations of the Galactic

Population of Black Hole X-Ray Binaries, AIP Conf. Proc. 1010, 404 (2008)

[5] Spitzermap, www.nasa.gov/centers/jpl/news/spitzera-20080603.html, (2008)

[6] Y. Tanaka, W.H.G. Lewin, Black Hole Binaries, in X-ray binaries, W.H.G. Lewin, J. van Paradijs, E.P.J. van den Heuvel (Eds), Cambridge, p. 126 (1996)

[7] N.E. White, J. van Paradijs, The Galactic Distribution of Black Hole Candidates in Low Mass X-ray Binary Systems, ApJ 473, L25 (1996) 